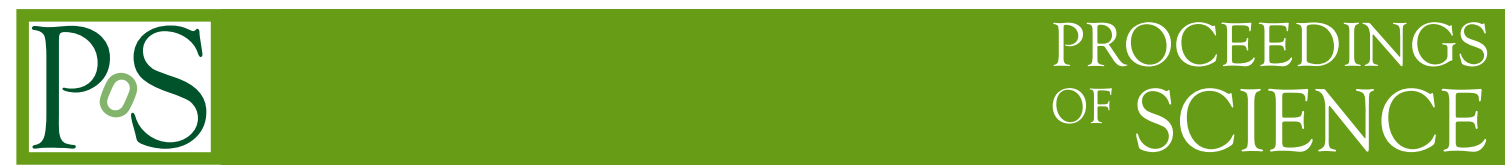

\title{
Underground nuclear astrophysics
}

\author{
Heide Costantini* \\ INFN Genova (IT) University of Notre Dame (USA) \\ E-mail: hcostantend.edu
}

\section{for the LUNA collaboration}

Cross section measurements for quiescent stellar $\mathrm{H}$ and $\mathrm{He}$ burning are hampered mainly by extremely low counting rate and cosmic background. Some of the main reactions of H-burning phase have been measured at the LUNA facility (Laboratory for Underground Nuclear Astrophysics) taking advantage of the very low background environment of the Underground Gran Sasso National Laboratory in Italy. An overview of the adopted experimental techniques will be given together with the latest results on the ${ }^{14} \mathrm{~N}(\mathrm{p}, \gamma){ }^{15} \mathrm{O}$ reaction and the status of the ongoing experiments.

International Symposium on Nuclear Astrophysics - Nuclei in the Cosmos - IX

25-30 June 2006

CERN

* Speaker. 


\section{H-burning in stars}

The fusion of hydrogen into helium represents the greater part of the stars life (main sequence stars) and is responsible for the prodigious luminosity of those stars. The basic concept of hydrogen burning is:

$$
4 p \rightarrow{ }^{4} \mathrm{He}+2 e^{+}+2 v+26.73 \mathrm{MeV}
$$

This transformation can occur through two different processes: the p-p chain and the CNO cycle. The sequence of reactions for the p-p chain is shown in fig 1 .

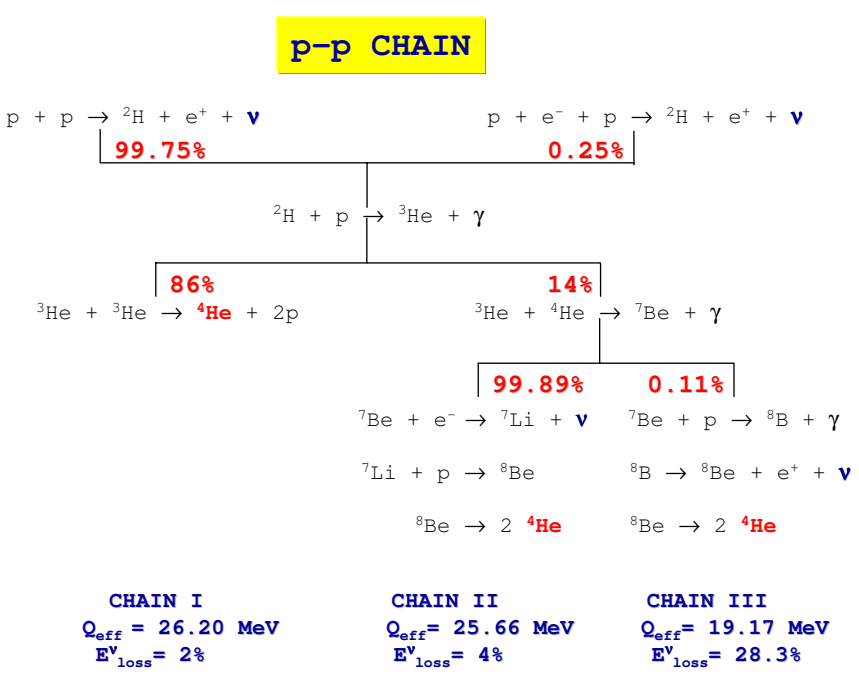

Figure 1: Scheme of the p-p nuclear reactions chain. The reactions are divided in three chains and the final result is the transformation of four protons into a helium nucleus.

When the center of a star reaches a temperature of $\mathrm{T} \approx 6 \times 10^{6} \mathrm{~K}$, the proton proton reaction rate becomes significant. The deuterium produced reacts with the proton sea producing ${ }^{3} \mathrm{He}$. At this point three different branches are possible. The ${ }^{3} \mathrm{He}+{ }^{3} \mathrm{He}$ reaction produces a ${ }^{4} \mathrm{He}$ nucleus and two protons (chain I):

$$
{ }^{3} \mathrm{He}+{ }^{3} \mathrm{He} \rightarrow{ }^{4} \mathrm{He}+\mathrm{p}+p
$$

An alternative is to catch an $\alpha$ particle:

$$
{ }^{3} \mathrm{He}+{ }^{4} \mathrm{He} \rightarrow{ }^{7} \mathrm{Be}+\gamma
$$

${ }^{7} \mathrm{Be}$ is unstable and decays via electron capture to ${ }^{7} \mathrm{Li}$ and a neutrino of either $0.38 \mathrm{MeV}$ or $0.86 \mathrm{MeV}$. Finally the ${ }^{7} \mathrm{Li}$ nucleus captures a proton producing ${ }^{8} \mathrm{Be}$, which decays producing two helium nuclei (chain II).

${ }^{7} \mathrm{Be}$ electron capture competes with proton capture producing ${ }^{8} \mathrm{~B}$, which decays to ${ }^{8} \mathrm{Be}$ producing a positron, a high energy neutrino $\left(0 \mathrm{MeV} \leq \mathrm{E}_{v} \leq 15 \mathrm{MeV}\right)$, and two helium nuclei (chain III). 
If in addition to hydrogen and helium, heavier elements are present in the star's interior, a second possibility for the conversion of hydrogen into helium is offered by a reaction cycle investigated in 1938 by H. Bethe and C.F. Von Weiszäker: the CNO-cycle. When the central temperature increases to $\mathrm{T} \approx 15 \times 10^{6} \mathrm{~K}$, carbon present in the star can react with the proton sea producing ${ }^{13} \mathrm{~N}$, which decays to ${ }^{13} \mathrm{C}$ which, in turn, captures another proton. As shown in equation 1.1 , at the end of this first part of the CNO-I cycle, four protons are transformed in one helium nucleus, exactly as in the p-p chain and with the same Q-value:

$$
\text { I CNO }\left\{\begin{aligned}
{ }^{12} C+p & \rightarrow{ }^{13} N+\gamma \\
{ }^{13} N & \rightarrow{ }^{13} C+e^{+}+v \\
{ }^{13} C+p & \rightarrow{ }^{14} N+\gamma \\
{ }^{14} N+p & \rightarrow{ }^{15} O+\gamma \\
{ }^{15} O & \rightarrow{ }^{15} N+e^{+}+v \\
{ }^{15} N+p & \rightarrow{ }^{12} C+\alpha \\
& \rightarrow{ }^{16} O+\gamma \quad 1 \%
\end{aligned}\right.
$$

The CNO cycle energy production rate increases faster with temperature than the $\mathrm{p}$-p chain reaction rate.

$$
\begin{aligned}
& \varepsilon_{n}(C N O)=\rho X Z_{C N O} T^{18} \\
& \varepsilon_{n}(p-p)=\rho X^{2} Z_{C N O} T^{4}
\end{aligned}
$$

where $\mathrm{X}$ is the hydrogen abundance and $\mathrm{Z}$ is the metal abundance. Therefore when the central stellar temperature exceeds $15 \times 10^{6} \mathrm{~K} \mathrm{H}$-burning occurs mainly through the $\mathrm{CNO}$ cycle.

Nuclear reactions during H-burning occur between charged particles and since the typical energy of the interacting nuclei $(\mathrm{KT} \approx \mathrm{keV})$ is much smaller than the Coulomb barrier, nuclear reactions occur through tunnelling effect. For charged particle reaction it is therefore possible to define the cross section as:

$$
\sigma(E)=\frac{S(E)}{E} e^{-2 \pi \eta}
$$

where the exponential term takes into account the tunnelling probability and the S-factor, $\mathrm{S}(\mathrm{E})$, smoothly varying function with the energy for non resonant reactions, includes all the nuclear properties of the reaction [1].

The reaction rate can thus be expressed as:

$$
<\sigma v>=\sqrt{\frac{8}{\pi \mu}} \frac{1}{(K T)^{3 / 2}} \int_{0}^{\infty} \frac{S(E)}{E} e^{-2 \pi \eta} e^{-E / K T} d E
$$

where $\mathrm{T}$ is the stellar temperature and $\phi(E) \propto e^{-E / K T}$ is the Maxwell-Boltzmann energy distribution that determines the velocity distribution of the nuclei inside the stellar plasma in the case of non-degenerate matter like in quiescent H-burning. 
The product of the two exponential terms leads to a well defined peak (the Gamow peak).

For a given stellar temperature T, nuclear reactions are taking place mainly inside the Gamow peak. In the case of $\mathrm{H}$-burning typical energies are of the order of tens of $\mathrm{keV}$ (for example 27 $\mathrm{keV}$ for ${ }^{14} \mathrm{~N}(\mathrm{p}, \gamma){ }^{15} \mathrm{O}$ and $22 \mathrm{keV}$ for $\left.{ }^{3} \mathrm{He}\left({ }^{3} \mathrm{He}, 2 \mathrm{p}\right){ }^{4} \mathrm{He}\right)$.

One of the goal of experimental nuclear astrophysics is to measure nuclear reactions at the energies at which they take place inside stars. Cross sections at the Gamow peak energy are of the order of $10^{-9}-10^{-12}$ barn corresponding to experimental counting rate ranging from few events per day to few events per month with typical laboratory conditions. The main problem in performing these reaction measurements at surface laboratory is that the detectors are continuously bombarded by cosmic rays, that interacting with the detector, the target and the surrounding materials, create background in the detectors. The cosmic background rate is generally much larger than the reaction rate at the Gamow peak.

Therefore experimentalists measure nuclear reactions at higher energies, transform the cross section into $\mathrm{S}$-factor and then extrapolate the $\mathrm{S}$-factor by means of different techniques (for example the R-matrix method [2]). However extrapolations can sometimes fail, for example in the case of an unpredicted narrow resonance at low energies or in the case of contributions from a subthreshold state. One solution to overcome this problems is to perform nuclear reaction measurements in an underground laboratory where the cosmic flux is reduced by several orders of magnitude.

\section{The LUNA project}

The Laboratory for Underground Nuclear Astrophysics (LUNA) has been designed to measure nuclear reactions mainly of $\mathrm{H}$-burning both of $\mathrm{p}$-p chain and $\mathrm{CNO}$ cycle at energies as close as possible to the Gamow peak. It is located deep underground in the Laboratori Nazionali del Gran Sasso (LNGS) in Italy. The Gran Sasso site is protected from cosmic rays by a rock cover (1400 $\mathrm{m}$ thick) equivalent to $3800 \mathrm{~m}$ water, suppressing the flux of cosmic ray induced muons by six orders of magnitude and the neutron flux by three orders of magnitude.

\subsection{Accelerators}

Since the main feature of an underground nuclear reaction measurement is the extremely low cross section, high beam current up to several hundreds of $\mu \mathrm{A}$ becomes a fundamental requirement. The LUNA facility operates a $50 \mathrm{kV}$ and a $400 \mathrm{kV}$ low energy electrostatic accelerators. Helium and proton beams are operated at currents of approximately $500 \mu \mathrm{A}$ for protons and 250 $\mu \mathrm{A}$ for $\alpha$. Measurements generally last several weeks and months and therefore long term energy stability becomes very important. Furthermore since the cross section depends exponentially from the energy (see eq. 1.4), an uncertainty of few per cent in the energy brings a very large error in the cross section determination. Therefore a good energy resolution is required. For the LUNA 400 $\mathrm{kV}$ accelerator a beam energy stability of $5 \mathrm{eV} /$ hour has been measured and the energy spread is of the order of $70 \mathrm{eV}[3]$.

\subsection{Main nuclear reaction studied at LUNA: experimental techniques and challenges.}

During the first phase of LUNA, the ${ }^{3} \mathrm{He}\left({ }^{3} \mathrm{He}, 2 \mathrm{p}\right)^{4} \mathrm{He}$ nuclear reaction has been studied using the home-made $50 \mathrm{kV}$ accelerator. The presence of a low energy resonance in the ${ }^{3} \mathrm{He}\left({ }^{3} \mathrm{He}, 2 \mathrm{p}\right){ }^{4} \mathrm{He}$ 
was invoked, before the SNO and Kamland results, as a possible nuclear explanation of the Solar Neutrino Problem.

A windowless gas target was used and the protons coming out from the reaction were detected with Si-detectors. Since cosmic background is suppressed in an underground laboratory, beam induced background can become a critical issue and has to be avoided by keeping a high level in target and beam purity. Gas targets are generally favored although impurities can still be present due for examples to vacuum pumps oil contamination. In the case of the ${ }^{3} \mathrm{He}\left({ }^{3} \mathrm{He}, 2 \mathrm{p}\right){ }^{4} \mathrm{He}$ a ${ }^{3} \mathrm{He}$ deuterium contamination in the gas target produced a very high background signal due to the strong ${ }^{3} \mathrm{He}(\mathrm{d}, \mathrm{p})^{4} \mathrm{He}$ reaction cross section. To distinguish between the protons coming from the ${ }^{3} \mathrm{He}\left({ }^{3} \mathrm{He}, 2 \mathrm{p}\right){ }^{4} \mathrm{He}$ reaction and the protons from the contaminant reaction, a coincidence between two silicon detectors was required as a signature of the emission of the two protons from the ${ }^{3} \mathrm{He}\left({ }^{3} \mathrm{He}, 2 \mathrm{p}\right){ }^{4} \mathrm{He}$ reaction. Thanks two this technique the background ${ }^{3} \mathrm{He}(\mathrm{d}, \mathrm{p}){ }^{4} \mathrm{He}$ reaction was completely suppressed [4].

The cross section was measured down to about $15 \mathrm{keV}$ covering all the solar Gamow peak and excluding the existence of any resonance. This important result showed that the solution of the Solar Neutrino Problem was not in the uncertainty of the Standard Solar Model.

After the success of this first experiment, a new 400KV accelerator was installed and the first measurement performed was the ${ }^{14} \mathrm{~N}(\mathrm{p}, \gamma){ }^{15} \mathrm{O}$ reaction.

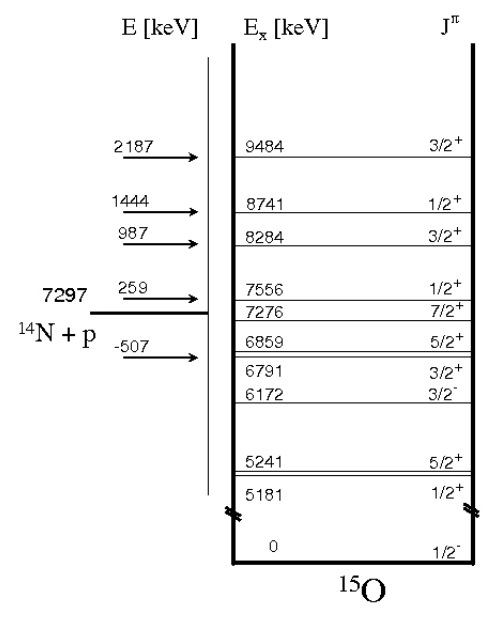

Figure 2: Level scheme of the ${ }^{15} \mathrm{O}$ nucleus.

The capture reaction ${ }^{14} \mathrm{~N}(\mathrm{p}, \gamma){ }^{15} \mathrm{O}$, the slowest process in the H-burning CNO cycle [1], is of high astrophysical interest as its reaction rate influences sensitively the age determination of globular clusters [5] and the solar neutrino spectrum [6, 7]. The capture cross section needs to be known down to $\mathrm{E}_{0}=30 \mathrm{keV}$ (the Gamow peak in core H-burning stars), which is far below the lowenergy limit of direct $\gamma$-ray measurements, i.e. the center-of-mass energy $\mathrm{E}=240 \mathrm{keV}$ [8]. Thus, the data had to be extrapolated over a large energy gap leading to a substantial uncertainty for the astrophysical S-factor at zero energy, $\mathrm{S}(0)$. According to the data and analysis of [8], there are two major and nearly equal contributions to $S(0)$ : the direct capture (DC) to the $6.79 \mathrm{MeV}$ state in ${ }^{15} \mathrm{O}$ and the capture to the ground state $(\mathrm{gs})$ in ${ }^{15} \mathrm{O}$ (see fig. 2). The latter process is enhanced 
due to a subthreshold resonance at $\mathrm{E}_{R}=-507 \mathrm{keV}$, the width of which was taken as a free parameter in the fit [8]. Subsequently, the data of Schröder et al [8] were reanalyzed by [9] using an Rmatrix approach. Contrary to the extrapolation by $[\overline{8}]$ for capture to the ground state, they reported a negligible contribution due to a smaller total width of the subthreshold resonance. A smaller width of the $6.79 \mathrm{MeV}$ state was supported by a lifetime measurement via Doppler-shift method [10] and by a Coulomb excitation measurement [11]. The LUNA collaboration started in 2001 a reinvestigation of ${ }^{14} \mathrm{~N}(\mathrm{p}, \gamma){ }^{15} \mathrm{O}$ studying this reactions in two different phases [12, 13, 14].

The measurement of the ${ }^{14} \mathrm{~N}(\mathrm{p}, \gamma){ }^{15} \mathrm{O}$ reaction is particularly well suited for an underground experiment since the Q-value of the reaction is $7.3 \mathrm{MeV}$.

As a matter of fact for $\gamma$-detectors, the advantage of an underground laboratory is particularly appreciated at $\gamma$-energies above $3 \mathrm{MeV}$ (see fig. 2.2). In this energy region the dominant background source are cosmic rays and by bringing the detector underground the background rate is reduced by more than three orders of magnitude. On the other hand at $\gamma$ energies below $3 \mathrm{MeV}$, the background spectrum is dominated by $\gamma$ radiation coming from environmental radioactive isotopes $\left({ }^{40} \mathrm{~K},{ }^{208} \mathrm{Tl},{ }^{214} \mathrm{Bi}\right.$ etc. $)$ that are always present in the rocks surrounding the laboratory. Radiative reaction measurements are consequently favored underground especially for high Q-value reactions.
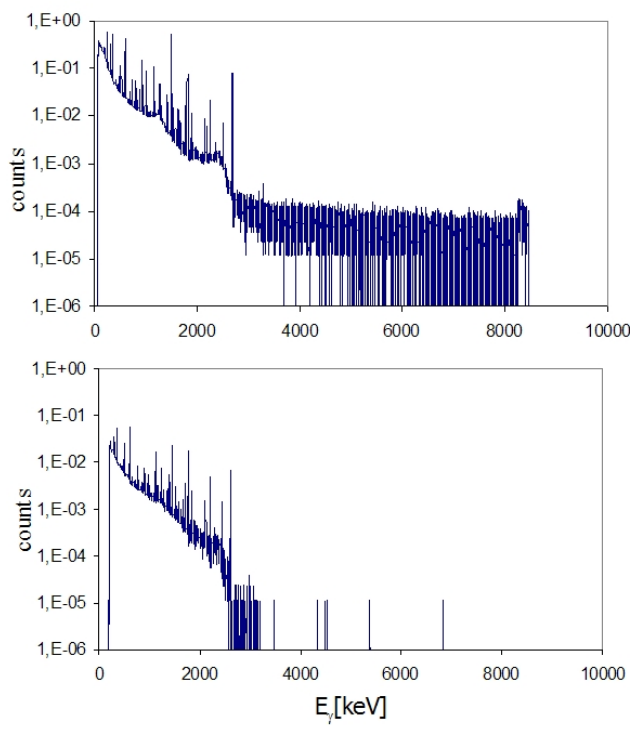

Figure 3: Background spectra taken with the $126 \%$ high purity germanium detector at surface (top spectrum) and underground with a $5 \mathrm{~cm}$ lead shielding (bottom spectrum). The measurement time is the same for both spectra and the counts are expressed in arbitrary units.

The goal of the first phase was to study the single $\gamma$-transitions and in particular the ground state transition. Therefore a solid target coupled with a high resolution HpGe-detector was used and it was possible to distinguish the single $\gamma$-decays. The ground state transition energy is close to the Q-value of the reaction and so a clean signal could be detected thanks to the background free energy spectra around $7 \mathrm{MeV}$ (fig. 2.2). In order to lower beam induced background, a careful study of different solid targets and backing materials was performed and TiN sputtered targets on a Ta backing were finally chosen. The spectrum of fig. 4 has been taken a $\mathrm{E}_{p}=180 \mathrm{keV}$. It 
clearly shows the contribution of both the studied reaction and of the parasitic background reaction ${ }^{11} \mathrm{~B}(\mathrm{p}, \gamma){ }^{12} \mathrm{C}$ due to Boron contaminant in Ta backing. Cross section measurements with solid target setup were performed in the energy range $\mathrm{E}_{b}=140-400 \mathrm{keV}$.

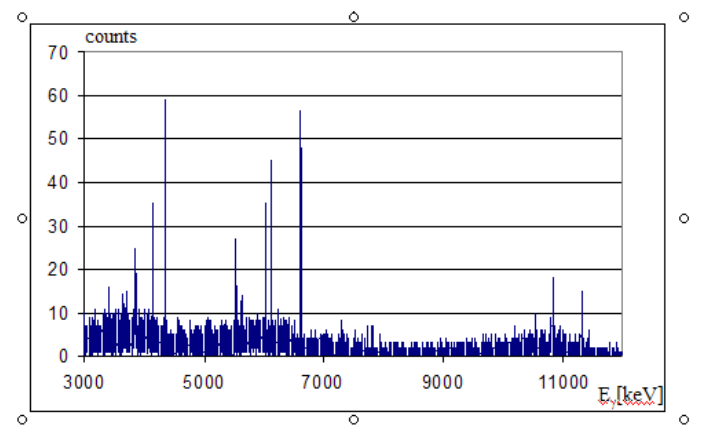

Figure 4: $\mathrm{HpGe}$ spectrum taken at $\mathrm{E}_{p}=180 \mathrm{keV}$. The secondary transitions from the ${ }^{14} \mathrm{~N}(\mathrm{p}, \gamma){ }^{15} \mathrm{O}$ reaction are visible together with the primary and secondary transitions from the ${ }^{11} \mathrm{~B}(\mathrm{p}, \gamma)^{12} \mathrm{C}$ reaction at $\mathrm{E}_{\gamma}=4.4$ and $11.6 \mathrm{MeV}$.

Beam induced background was mainly disturbing the measurements at intermediate energies, since by decreasing the beam energy, the Coulomb barrier was sensitively affecting the cross section of the parasitic reactions.

The weak side of using a high resolution germanium detector is the relatively low detection efficiency. Therefore to push the cross section measurements toward lower energies a second phase of the experiment was started using a nearly $4 \pi$ BGO summing crystal. All the $\gamma$-cascades are summed together to a peak at $\mathrm{E}_{\gamma}=\mathrm{Q}+\mathrm{E}_{c m}$ around $7 \mathrm{MeV}$ where the detection efficiency is about $65 \%$ [14]. Due to the intense beam current $\left(\mathrm{I}_{p}=500 \mu \mathrm{A}\right)$, the gas target local density along the beam path is decreased [15]. A careful study of beam heating effect was performed and the results were implemented in the final data analysis [16]. In the gas target experiment the main source of beam induced background was coming from impurities in the collimators and the beam stop. By replacing the $\mathrm{N}$ gas with the inert ${ }^{4} \mathrm{He}$ gas, beam induced background measurements could be performed and the obtained spectra subtracted to the ${ }^{14} \mathrm{~N}$ spectra. Again beam induced background was a major problem at intermediate energies. At the lowest measured energy $\left(\mathrm{E}_{b}=80 \mathrm{keV}\right)$ the main background source in the ROI was coming from $(\mathrm{n}, \gamma)$ reactions from neutrons produced either by $(\alpha, n)$ from natural radioactivity or residual cosmic muons.

The two different approaches were complementary and both took extreme advantage of the low background laboratory (see figure 5 ).

The final results from both experiments were in good agreement with new measurement by [17. 18] but differed in the weight of the contributions from the various transitions. The extracted stellar reaction rate confirmed the conclusion of [9] that the rate has to be reduced by nearly a factor of two at low temperatures, but it is in good agreement with NACRE [19] above $\mathrm{T}_{6}=150$. In conclusion, with the present determination of the reaction rates the main astrophysical consequence is the age increase of the Globular Clusters by about 1 Gyear, i.e about $14 \pm 1$ Gyears depending by the metallicity of the Globular Cluster and the reduction of a factor 2 of solar CNO neutrinos.

In the case of low Q-value reactions, the advantage of an underground laboratory is not evident 


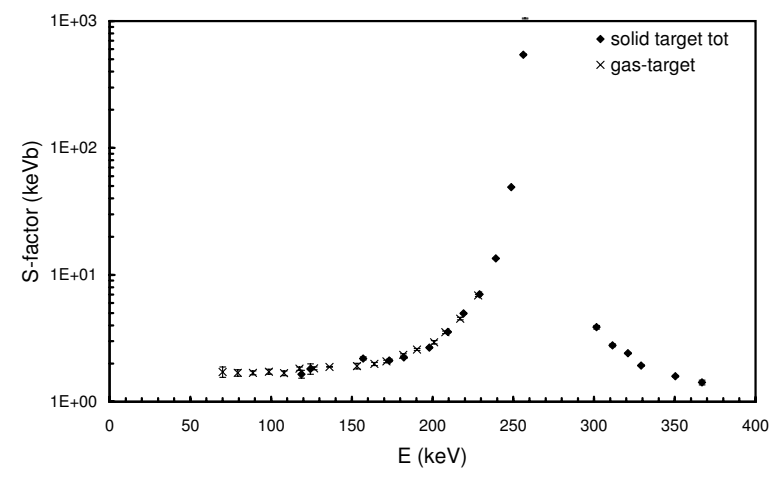

Figure 5: Comparison between the astrophysical factor obtained with the LUNA high resolution and high efficiency experiments for the ${ }^{14} \mathrm{~N}(\mathrm{p}, \gamma){ }^{15} \mathrm{O}$ reaction.

at first sight. Environmental background is also present underground. Detectors can be shielded passively with proper Lead and Copper shield as on surface. However there is a big advantage in a underground laboratory. In a surface laboratory passive shielding can be built around the detectors but above a certain thickness the shield efficiency cannot be increased by adding further shield material since cosmic muons interact with the shielding material and can create background signals in the detector. Obviously this problem is dramatically reduced in an underground laboratory.

The ${ }^{3} \mathrm{He}\left({ }^{4} \mathrm{He}, \gamma\right){ }^{7} \mathrm{Be}$ has a Q-value of $1.6 \mathrm{MeV}$. This reaction is presently under study at LUNA. The ${ }^{3} \mathrm{He}(\alpha, \gamma){ }^{7} \mathrm{Be}$ reaction is one of the major source of uncertainty in determing the Boron solar neutrino Flux and dominates over the present observational accuracy $\Delta(\Phi(B)) / \Phi(B)=7 \%$ [6]. The foreseeable accuracy of the new generation solar neutrino experiments is $\Delta(\Phi(B)) / \Phi(B)=3 \%$. This result could illuminate about solar physics if the uncertainty on $S_{34}$ is reduced to a corresponding level. Moreover this reaction plays an important role in understanding the primordial ${ }^{7} \mathrm{Li}$ abundance [20]. Past measurements, that go back to twenty years ago, have been performed using two different methods. In the first method prompt $\gamma$-rays $\left(\mathrm{E}_{\gamma} \approx 1.6\right.$ and $\left.1.2 \mathrm{MeV}\right)$, coming from direct $\alpha$-capture, are detected, while in the second one the delayed $\gamma_{\mathrm{s}}\left(\mathrm{E}_{\gamma}=478 \mathrm{keV}\right)$, coming from ${ }^{7} \mathrm{Be}$ decay through electron capture, are counted.

A global analysis indicates that the extrapolated $S(0)$ obtained with the activation method is sistematically $13 \%$ higher than the prompt- $\gamma$ result. A recent activation study [21] reduces the discrepancy to $9 \%$ still not at the precision level of the ${ }^{8} \mathrm{~B}$ neutrino data.

The goal of the experiment at LUNA is to measure the cross section of the reaction using both techniques at the same time reducing the error on the astrophysical factor $S(3,4)$ to $4 \%$.

The prompt capture $\gamma$-rays are measured with an ultra-low 135\% background germanium detector heavily shielded $\left(0.3 \mathrm{~m}^{3}\right.$ of Lead and Copper) and placed at close distance to a ${ }^{3} \mathrm{He}$ windowless gas target. The suppression factor obtained with the shield for $\gamma \mathrm{s}$ below $2 \mathrm{MeV}$ is of five orders 
of magnitude (see fig. 6).

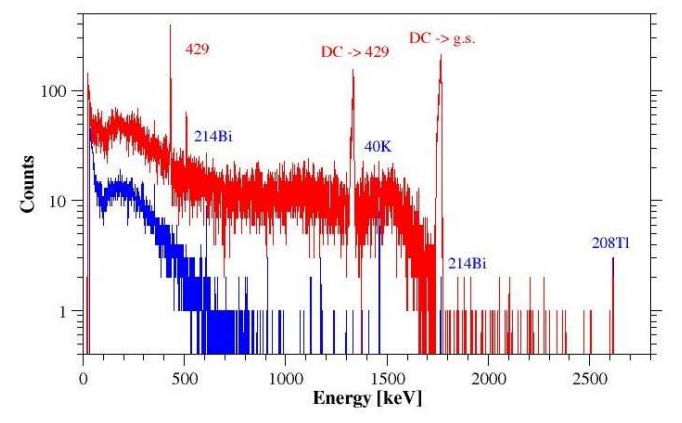

Figure 6: Comparison between the background spectrum and the reaction spectrum at $\mathrm{E}_{\alpha}=400 \mathrm{keV}$ obtained with the ultra low background $135 \%$ HpGe detector. The suppression factor due to the passive $\mathrm{Pb}-\mathrm{Cu}$ shield of the natural environmental radioactivity is of five orders of magnitude.

Besides from the rocks, environmental background can come also from all the materials surrounding the detector and from the detector itself. Therefore the target chamber and all the setup close to the detector were made of OFC copper and no welding materials have been used in the chamber assembling. The ${ }^{7} \mathrm{Be}$ nuclei are collected on the beam stop and are counted off-line by a $125 \% \mathrm{HpGe}$ detector, completely shielded by $15 \mathrm{~cm}$ of lead and $10 \mathrm{~cm}$ of copper on each side, positioned in the low activity laboratory at LNGS. The typical beam current is about $250 \mu \mathrm{A}$ and therefore to avoid systematic uncertainties due to beam heating effects the target density is measured through $\alpha$-Rutherford scattering cross section with a silicon detector positioned inside the target chamber.

When the ${ }^{3} \mathrm{He}(\alpha, \gamma){ }^{7} \mathrm{Be}$ reaction measurement is finished, LUNA will start to study the ${ }^{25} \mathrm{Mg}(\mathrm{p}, \gamma)^{26} \mathrm{Al}$ reaction. It's the slowest reaction of the $\mathrm{Mg}$-Al cycle. The $\beta^{+}$decay of ${ }^{26} \mathrm{Al}_{g s}$ to the excited state of ${ }^{26} \mathrm{Mg}$ gives rise to a $1.8 \mathrm{MeV} \gamma$-ray, one of the most important line for $\gamma$-astronomy [22]. The level scheme of ${ }^{26} \mathrm{Al}$ is very complicated and a lot of resonances of low intensities are present in the astrophysical energy region. The measurement of the weak low energy resonances will be performed at LUNA coupling a high efficient $4 \pi$ BGO summing crystal with a high purity ${ }^{25} \mathrm{Mg}$ solid target. First test of target purity and stability has been performed.

\section{Outlook}

When the measurement of the ${ }^{25} \mathrm{Mg}(\mathrm{p}, \gamma){ }^{26} \mathrm{Al}$ reaction is finished, LUNA will end the current scientific approved program.

In September 2005 a working group has been formed inside the LUNA collaboration with the goal to determine a list of reaction of astrophysical relevance that could be studied at the $400 \mathrm{kV}$ accelerator and for which an underground approach represents a clear advantage. An other goal of the working group is to investigate the importance and the possibility of the installation of a higher energy accelerator that could be used for the study of He-burning key nuclear reactions.

At the same time a new idea of an underground accelerator facility to be installed inside the future DUSEL underground laboratory is developing in the United States nuclear astrophysics co- 
munity. The new facility (ALNA: Accelerator Laboratory for Nuclear Astrophysics Underground) should be mainly focused on the study of He-burning and C-burning reactions. ALNA will couple the low background environment of an underground laboratory with state of the art detection techniques aimed to maximum detection efficiency and unique event identification capability for active background reduction. The idea is to install in a first phase a small accelerator for light ions to study $(\alpha, n)$ and $(\alpha, \gamma)$ reactions in forward kinematics, and in a second phase a higher energy heavy ion machine to study proton and $\alpha$ capture reactions in inverse kinematics using the recoil separator technique.

\section{References}

[1] C. Rolfs and W.S. Rodney: Cauldrons in the cosmos, University of Chicago Press (1988)

[2] A.N. Lane and R.G. Thomas, Rev. Mod. Phys. 30(1958)257

[3] A. Formicola et al., Nucl. Inst. Meth. A, 507(2003)609

[4] R. Bonetti et al.,Phys. Rev. Lett. 82(1999)5205

[5] G. Imbriani et al, A\&A, 420(2004)625

[6] J. N. Bahcall and M. H. Pinsonneault, Phys. Rev. Lett. 92(2004)121301

[7] S. Degl'Innocenti et al., Phys. Lett. B 590(2004)13

[8] U. Schröder et al, Nucl. Phys. A 467(1987)240

[9] C. Angulo and P. Descouvemont, 2001, Nucl. Phys. A 690(2001)755

[10] P.F. Bertone et al, Phys. Rev. Lett. 87(2001)152501

[11] K. Yamada et al, Phys. Lett. B 579(2004)265

[12] A. Formicola et al, Phys.Lett.B, 591(2004)61-68

[13] G. Imbriani et al, Eur.Phys.Journal A, 25(2005)455-466

[14] A. Lemut et al., Phys. Lett. B 634(2006)483-487

[15] J. Görres et al., Nucl. Inst. Meth. A, 177(1980)295

[16] D. Bemmerer et al., submitted to Nucl. Phys. A

[17] A.M. Mukhamedzhanov et al, Phys. Rev. C 67(2003)065804

[18] R.C. Runkle et al.,Phys. Rev. Lett. 94(2005)082503

[19] C. Angulo et al, Nucl. Phys. A 656(1999)3

[20] A. Coc et al., Astrophys. J. 600(2004)544

[21] B.S. Nara Singh et al., Phys. Rev. Let. 93(2004)262503

[22] R. Diehl et al. A\&A, 298(1995)445. 